DOI:Registering DOI

\title{
Remote Sensing Image Recognition Based on Multi-attention Residual Fusion Networks
}

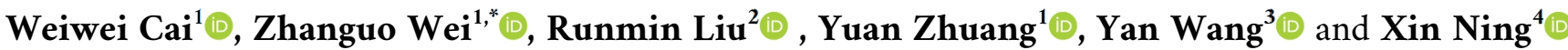 \\ ${ }^{1}$ Central South University of Forestry and Technology, Changsha 410004, China \\ ${ }^{2}$ Wuhan Sports University, Wuhan 430079, China \\ ${ }^{3}$ Changsha Astra Information Technology Co., Ltd. Changsha 410219, China \\ ${ }^{4}$ Institute of Semiconductors, Chinese Academy of Sciences, Beijing 100083, China \\ * Corresponding author: Zhanguo Wei (t20110778@csuft.edu.cn)
}

Manuscript Revised 20 April 2021; Accepted 20 April 2021; Published 21 April 2021

Academic Editor: Liang Liu

\begin{abstract}
Since each sample in a hyperspectral remote sensing image is made up of high-dimensional features and contains a wealth of remote sensing features, feature selection and mining become more difficult. To address this issue, a multi-attention residual integrated network (MARB-Net) algorithm is proposed, which reduces redundant features while increasing feature fusion and, as a result, improves hyperspectral image recognition. First, assign multiple weights to each feature using multiple attention mechanism models; then, deep mine and integrate the features using the residual network; and finally, perform contextual semantic integration on the deep fusion features using the Bi-LSTM network. The recognition task should be completed by the Softmax classifier. The experimental results on three multiclass public data sets show that the MARB-Net algorithm proposed in this paper is effective.
\end{abstract}

Index Terms: Attention mechanism, Residual network, Neural networks, and Remote sensing image.

\section{Introduction}

Hyperspectral images (HSIs) from remote sensing are made up of high-dimensional features. These characteristics describe each sample in the image in great detail, making hyperspectral research easier to categorize. Ocean remote sensing[1]-[15], desert vegetation[16], urban division[17], and other fields use hyperspectral remote sensing. The rapid development of deep learning $[14][19]$ in recent years has accelerated the classification and recognition efficiency of hyperspectral data while also improving recognition accuracy. Feature selection and deep mining are required for removing redundant features and retaining more deep features, as well as ensuring that highdimensional image classification and recognition accuracy improves. $\mathrm{Wu}$ Chen et al. [5] used a descriptive dictionary learning method to calculate the sparse coefficients of image features, perform feature selection, and merge the features of each image, improving transfer recognition learning of new types of scenes and proving the method's effectiveness. By combining the high-level feature selection of different CNNs, Ge Yun et al. [6] were able to preserve a large number of deep features, eliminate redundant features using maximum pooling and principal component analysis algorithms, and finally show that the method extracts feature retrieval. El-Shafie et al. [7] used CNN to eliminate shallow redundant features in the original image, improve computational efficiency while retaining a large number of deep features, and finally improve recognition accuracy. Fuding Xie [8] used a five-fold crossvalidated support vector machine to perform feature deep mining and hyperspectral image classification after calculating the correlation coefficients of adjacent bands to select the appropriate band subset. based on two publicly available databases Demonstrate the algorithm's efficiency. Machine learning and deep learning both play a role in removing redundant features and mining deep features, as demonstrated by the methods above. As a result, building a deep network to remove redundant features and mine more deep features is possible.

In recent years, deep learning is making more and more progress in image classification [12] as a result of its powerful feature mining and learning capabilities. Wen Ma et al. [18] proposed a residual neural network (Res -Net) that predicts components of different frequencies while simplifying deep network training, increasing network flexibility, and saving memory; Yiming Zhou et al. [9] used the Alex-Net network 
model combined with the weight transfer algorithm to train spatial remote sensing images, and finally greatly improved the classification accuracy; The NWPU-RESISC45 data set has been subjected to a large number of simulation experiments, all of which have yielded positive results. Zhang J et al. [10] proposed an efficient dense network (Dense-Net), which eliminates a large number of training parameters of the algorithm and deepens the number of network layers, and obtains more abstract semantic information, and finally passes UCM Data set, AID data set and NWPU-RESISC45 data set prove the feasibility of the algorithm. To assign weights to remote sensing image features, You Hongfeng et al. [2] developed an integrated algorithm based on attention and a separate recurrent neural network. Experiments have shown that the attention mechanism [11] assigns more weight coefficients to useful features, eventually eliminating a large number of redundant features while retaining more deep features, and that independent recurrent neural networks can improve Classification accuracy of remote sensing images by integrating with the contextual semantic information of deep features.

We discovered that different models can mine different deep features based on the research mentioned above. We propose a residual integrated network based on multi-attention to achieve this goal (MARB-Net). Multiple attention mechanism algorithms are used to assign different weights to each feature. Simultaneously, we've introduced multiple residual network model blocks in order to better mine and fuse deep features. The final contextual semantic integration features were obtained by integrating the deep features through a two-way long and short-term memory network. Finally, while mining deep features, the MARB-Net network eliminates a large number of redundant features, and the deep features gain more contextual semantic integration features thanks to the integration algorithm. The algorithm can better mine the features of high-dimensional hyperspectral images, according to experimental results.

The main contributions of this article are as follows:

1) This paper proposes a multi-attention mechanism that assigns multiple weights to the original hyperspectral features, allows each feature to be distinguished to the greatest extent possible, and ensures that redundant features are removed.

2) This paper uses a residual network to eliminate redundant features and improve deep feature mining and fusion.

3) This paper employs a Bi-LSTM network to integrate deep features mined by convolution kernels of various scales in order to better integrate the context semantics of deep features.

4) The results of the experiments on three public data sets, Indian, Pavia, and Salinas, were visualized in this article, demonstrating the effectiveness of the MARB-Net algorithm.

The following is a summary of the rest of the paper: The MARB-Net method is introduced in the second part; the experimental results are analyzed in the third part; and the conclusion is presented in the fourth part.

\section{Methodology}

The MARB-Net algorithm's overall model framework is shown in Figure 1. The multi-attention mechanism is used to obtain full feature mining; the residual network is then used to calculate the residual of the multi-attention mechanism in order to generate more representative residual features; and finally, the two-way long and short-term memory network is used to implement the integrated algorithm of deep features. Finally, the Softmax classification function predicts each pixel, and the final recognition is achieved.

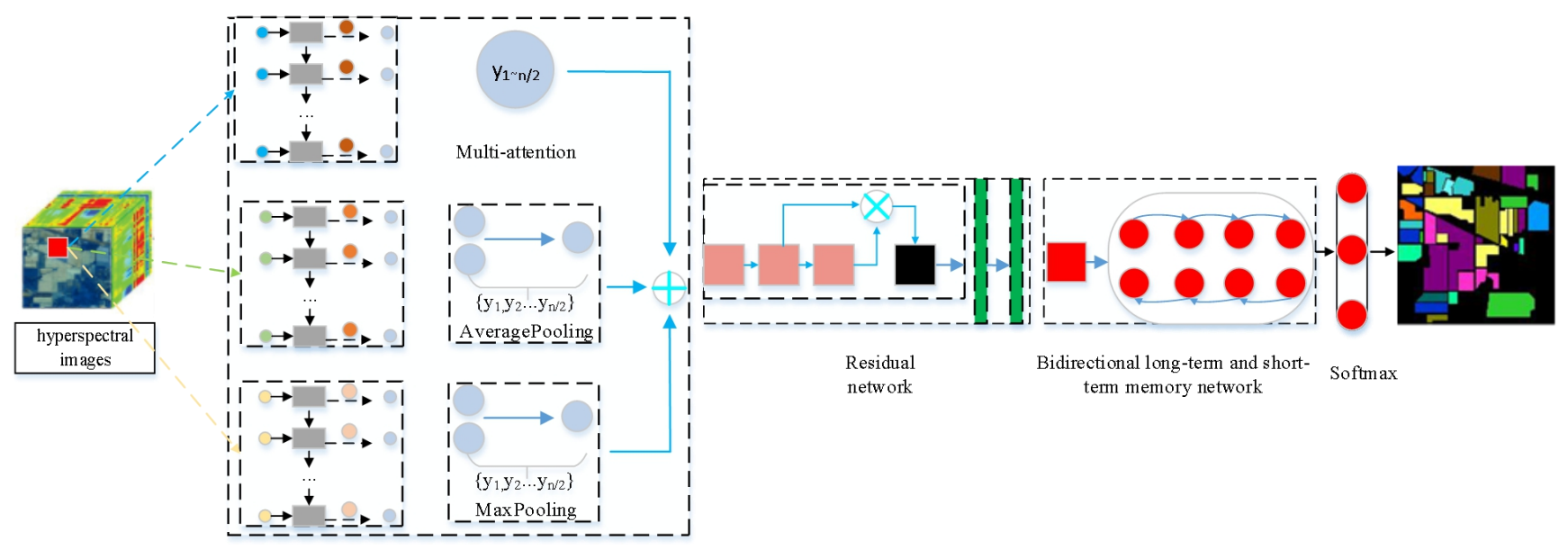

Fig.1. MARB-Net algorithm model diagram. Multi-attention represents the multi-attention mechanism model; Residual network represents the residual network, and the green bar represents a group of sub-modules; $\oplus$ represents the feature fusion operation; $\otimes$ represents the calculation of the sum of feature residuals; the pink square represents the convolution operation; the black square represents the average pooling operation.

\subsection{Multi-attention mechanism}

The attention mechanism works on the basis of a probability weight distribution. This mechanism calculates the features at different points in time, giving the features with more information a higher weight value and thus improving the quality of the hidden layer's high-dimensional features. 


$$
c_{i}=\sum_{j=1}^{n} \frac{\exp \left(e_{i, j}\right)}{\sum_{k=1}^{n} \exp \left(e_{i k}\right)} h_{j}
$$

Where $h_{j}$ is the information of the hidden layer, $e$ represents the energy value at the first moment, and $c_{i}$ represents the context generated by these $i$ points.

The multi-attention mechanism proposed in this paper is composed of three attention mechanisms. The original attention mechanism retains half of the higher-weighted features; the average pooling attention mechanism, which primarily uses the tie pooling function to retain local features; and the maximum pooling attention mechanism, which retains all of the higher-weighted features. Multiple attention mechanisms assign multiple weights to the original hyperspectral features to maximize the distinction between features and provide for the removal of redundant features later Guaranteed; the attention mechanism is primarily used to highlight the local features of the attention mechanism with larger weights; Figure 1 depicts the multi-attention implementation process in detail. The joint output formula of multi-attention mechanism features is as follows:

$$
F_{(o, a, m)}=\text { Concatenate }(o \oplus a \oplus m)
$$

After calculating and assigning weights through the attention mechanism formula (1), through three processing weighting algorithms, three features with new weights are generated. Among them, $O$ represents the output of the original attention mechanism feature; $a$ represents the output of the maximum pooling attention mechanism feature; $m$ represents the output of the average pooling attention mechanism feature. $F(o, a, m)$ represents the joint output of multiple attention features. $\oplus$ Indicates the calculation of the feature fusion algorithm. The fusion features output by the fusion of multiple attention mechanisms can provide richer shallow features for the subsequent residual network.

\subsection{Residual network}

We chose a residual network to better eliminate redundant features and mine and fuse deep features. The difference between a residual network and a regular convolutional network is that the residual network uses a jump-type residual operation to reduce the characteristic parameters while avoiding the gradient disappearance and degradation issues that the deep network causes. The formula for calculating the residual network is shown below.

$$
Y=W_{i} \delta\left(W_{i-1} X_{i-1}\right)+X
$$

where $Y$ represents the output of the $i$-th layer; $W$ represents the weight matrix; $\delta$ represents the Relu activation function; $X$ represents the input of the current layer. Through the residual network, we not only eliminate redundant features, but also conduct deeper feature mining and fusion of highdimensional remote sensing images, and at the same time solve the problem of gradient disappearance and explosion in deep networks.

\subsection{Bi-LSTM network}

The Bi-LSTM network is chosen in this paper to realize the information integration of the residual network's deep features. The contextual semantic features between the deep features are contained in the time series features generated by Bi-LSTM, which strengthen the correlation between the deep features. Softmax is then used to classify and identify it. Figure 2 depicts the Bi-LSTM network.

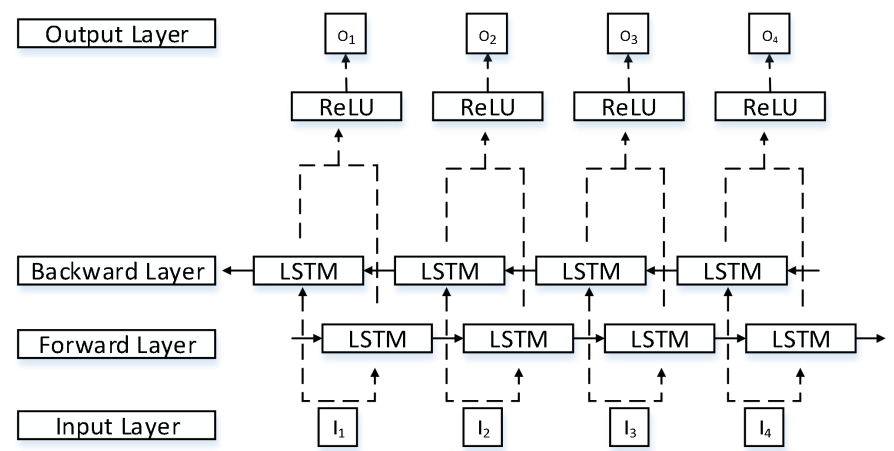

Fig.2. Input Layer represents the deep features of the residual network output Output Layer represents the contextual semantic features after deep feature integration; Forward Layer represents the forward propagation of long and short-term memory; Backward Layer represents the back propagation of long and short-term memory; ReLU Represents the nonlinear activation function.

\section{Experiments}

\subsection{Datasets and evaluation methods}

We evaluate our proposed algorithm using three publicly available hyperspectral data sets in this paper. Indina Pines is the first data set, with a total number of sample points in the image of 21025 and a total feature dimension of 200 for each sample point. There are 16 foreground classes and 1 background class in the entire image. Pavia University is the second data set, with a total of 207,400 sample points in the image and a feature dimension of 103 for each sample point. There are nine foreground classes and one background class in the entire image. The third data set is Salinas, which has 111104 sample points, a feature dimension of 115 for each sample point, and a total of 16 foreground classes and 1 background class. Finally, the performance of each classification algorithm is assessed using three indicators: total classification accuracy (OA), average classification accuracy (AA), and Kappa coefficient.

The calculation formula of Overall Accuracy (OA) is as follows:

$$
O A=\frac{T P}{T N}
$$

where $T P$ represents the number of correctly classified images in the image dataset, and $T N$ represents the total number of images in the image data set.

The calculation formula of Average Accuracy (AA) is as follows:

$$
A A=\frac{1}{c} \sum_{i=1}^{c}(x)
$$


where $c$ is the number of categories, and $x$ is the percentage of correctly classified pixels in a single category. Finally, the calculation formula of Kappa coefficient is:

$$
\text { Kappa }=\frac{\mathrm{OA}-\mathrm{P}_{e}}{1-\mathrm{P}_{e}}
$$

$P_{e}$ is defined as:

$$
P_{\mathrm{e}}=\frac{a_{1} \times b_{1}+a_{2} \times b_{2}+a_{3} \times b_{3} \ldots+a_{n} \times b_{n}}{T N^{2}}
$$

where $a_{1}, a_{2}, a_{3}, \ldots, a_{n}$ represents the true number of samples of each type, and $b_{1}, b_{2}, b_{3}, \ldots, b_{n}$ represents the predicted number of samples of each type.

\subsection{Hyperparameter settings}

The model algorithm's classification and recognition effects are directly influenced by hyperparameter settings. When the parameters are updated, this article uses a simple and efficient Adam optimizer that is unaffected by the gradient expansion change. The experiments with all the models were performed on a computer equipped with a single NVIDIA GTX1080 GPU ( $8 \mathrm{~GB})$, and the other environmental settings and training set division methods were identical. We used the Adam optimizer with a learning rate of 0.01 . The attenuation rate of the firstorder matrix was 0.9 , and the attenuation rate of the second- order matrix was 0.999. Epsilon was set to $1 \mathrm{e}-08$ instead of to 0 to prevent the denominator from being 0 ; We batch processed 1,000 samples each time. we repeated 10 times for each group of experiments and selected the best group of experiments.

Table 1 Hyperparameter settings

\begin{tabular}{c|c}
\hline Type & Hyperparameters \\
\hline \multirow{4}{*}{ Adam } & $\operatorname{lr}=0.0001$ \\
\cline { 2 - 2 } & bata_1 $=0.9$ \\
\cline { 2 - 2 } & bata_2 $=0.999$ \\
\cline { 2 - 2 } & Epsilon $=1^{\mathrm{e}-08}$ \\
\cline { 2 - 2 } & Decay $=3^{\mathrm{e}-8}$ \\
\hline
\end{tabular}

\subsection{Comparison of the results of the experiment}

By learning different deep features, different models produce different recognition results. To ensure a fair comparison, all experiments are carried out in the same environment and with the same parameters. The proposed MARB-Net model is compared to four different models: Alex-Net [9], Res-Net [18], Dense-Net [10], and ATGIR [2]. The following table displays the results of the various data sets (Table 2 is the Indian data set; Table 3 is the Pavia University data set and Table 4 is the Salinas data set

Table 2 Extract 5\%,20\%, 40\% of the Indian data set samples as the training set to achieve classification, where the boldface represents the result of the improved algorithm

\begin{tabular}{|c|c|c|c|c|c|c|c|c|c|}
\hline \multirow{2}{*}{ Methods } & \multicolumn{3}{|c|}{$5 \%$} & \multicolumn{3}{|c|}{$20 \%$} & \multicolumn{3}{c|}{$40 \%$} \\
\cline { 2 - 10 } & OA & AA & Kappa & OA & AA & Kappa & OA & AA & Kappa \\
\hline Res-Net[18] & 0.7127 & 0.6834 & 0.6727 & 0.8257 & 0.8156 & 0.8008 & 0.9110 & 0.8967 & 0.8985 \\
\hline Dense-Net[10] & 0.6732 & 0.6569 & 0.6252 & 0.7783 & 0.7885 & 0.7461 & 0.8900 & 0.8811 & 0.8745 \\
\hline Alex-Net[9] & 0.7124 & 0.6829 & 0.6686 & 0.8020 & 0.8152 & 0.7752 & 0.8901 & 0.9001 & 0.8744 \\
\hline ATGIR[2] & 0.7163 & 0.6917 & 0.6787 & 0.7903 & 0.8303 & 0.8061 & 0.8916 & 0.9069 & 0.8937 \\
\hline MARB-Net & $\mathbf{0 . 7 4 6 1}$ & $\mathbf{0 . 7 3 7 5}$ & $\mathbf{0 . 6 9 7 8}$ & $\mathbf{0 . 8 7 3 5}$ & $\mathbf{0 . 8 5 7 5}$ & $\mathbf{0 . 8 5 5 6}$ & $\mathbf{0 . 9 2 5 9}$ & $\mathbf{0 . 9 1 4 0}$ & $\mathbf{0 . 9 1 5 4}$ \\
\hline
\end{tabular}

Table 3 Extract 1\%, 10\%, and 20\% of the Pavia data set samples as the training set to achieve classification, where the boldface represents the result of the improved algorithm

\begin{tabular}{|c|c|c|c|c|c|c|c|c|c|}
\hline \multirow{2}{*}{ Methods } & \multicolumn{3}{|c|}{$1 \%$} & \multicolumn{3}{|c|}{$10 \%$} & \multicolumn{2}{c|}{$20 \%$} \\
\cline { 2 - 10 } & OA & AA & Kappa & OA & AA & Kappa & OA & AA & Kappa \\
\hline Res-Net[18] & 0.8636 & 0.8399 & 0.8176 & 0.9484 & 0.9445 & 0.9314 & 0.9619 & 0.9549 & 0.9494 \\
\hline Dense-Net[10] & 0.8436 & 0.8349 & 0.8146 & 0.9345 & 0.9266 & 0.9130 & 0.9525 & 0.9491 & 0.9369 \\
\hline Alex-Net[9] & 0.8724 & 0.8538 & 0.8298 & 0.9437 & 0.9348 & 0.9252 & 0.9597 & 0.9495 & 0.9466 \\
\hline ATGIR[2] & 0.8755 & 0.8339 & $\mathbf{0 . 8 3 5 5}$ & 0.9484 & 0.9395 & 0.9316 & 0.9578 & 0.9465 & 0.9440 \\
\hline MARB-Net & $\mathbf{0 . 8 7 6 3}$ & $\mathbf{0 . 8 7 0 6}$ & 0.8341 & $\mathbf{0 . 9 5 2 4}$ & $\mathbf{0 . 9 4 6 2}$ & $\mathbf{0 . 9 3 6 8}$ & $\mathbf{0 . 9 6 4 4}$ & $\mathbf{0 . 9 5 6 1}$ & $\mathbf{0 . 9 5 2 7}$ \\
\hline
\end{tabular}

Table 4 Extract $1 \%, 10 \%$, and $20 \%$ of the Salinas data set samples as the training set to achieve classification, where the boldface represents the result of the improved algorithm

\begin{tabular}{|c|c|c|c|c|c|c|c|c|c|}
\hline \multirow{2}{*}{ Methods } & \multicolumn{3}{|c|}{$1 \%$} & \multicolumn{3}{c|}{$10 \%$} & \multicolumn{2}{c|}{$20 \%$} \\
\cline { 2 - 10 } & OA & AA & Kappa & OA & AA & Kappa & OA & AA & Kappa \\
\hline Res-Net[18] & 0.8963 & 0.9223 & 0.8756 & 0.9557 & 0.9790 & 0.9506 & 0.9665 & 0.9842 & 0.9627 \\
\hline Dense-Net[10] & 0.8805 & 0.9084 & 0.8672 & 0.9381 & 0.9649 & 0.9310 & 0.9607 & 0.9795 & 0.9562 \\
\hline Alex-Net[9] & 0.8894 & 0.9228 & 0.8767 & 0.9525 & 0.9740 & 0.9471 & 0.9661 & 0.9828 & 0.9623 \\
\hline ATGIR[2] & 0.8985 & 0.9297 & 0.8872 & 0.9518 & 0.9749 & 0.9464 & 0.9682 & 0.9842 & 0.9646 \\
\hline MARB-Net & $\mathbf{0 . 9 1 2 6}$ & $\mathbf{0 . 9 4 8 3}$ & $\mathbf{0 . 9 0 0 1}$ & $\mathbf{0 . 9 5 6 4}$ & $\mathbf{0 . 9 7 8 2}$ & $\mathbf{0 . 9 5 1 4}$ & $\mathbf{0 . 9 6 9 5}$ & $\mathbf{0 . 9 8 5 1}$ & $\mathbf{0 . 9 6 6 0}$ \\
\hline
\end{tabular}

1) Results of the Indian data set: The Indian data set is a small sample data set. We use $5 \%, 20 \%$, and $40 \%$ of the total sample data as the training set. It can be clearly seen from Table 2 that the average accuracy (AA) of various samples of the algorithm with the attention mechanism is greater than that of the algorithm without the attention mechanism, because the attention mechanism assigns more information to the features in a small sample The larger weight causes the ATGIR algorithm to be $0.88 \% \sim 3.48 \%$ higher than the other three groups of model algorithms without attention mechanism. With the improvement of training samples, the segmentation results 
of each model have been improved, but the effect of the algorithm including the attention mechanism is better, an increase of $0.58 \% \sim 2.68 \%$. At the same time, the overall residual network experimental results are better than dense network and Alex-Net, because the residual retains a large number of shallow features, while the residual calculation better integrates the deep features, and obtains more features for the final recognition, So that the overall performance of retaining features is stronger, and the prediction results of the other two models without attention mechanism are improved by $1 \%$ to $2.09 \%$. At the same time, it can be clearly seen from the experimental results of the kappa coefficient that the residual network has better performance in mining features. And our proposed multi-attention residual ensemble network on the same training sample is in OA (higher than the other four groups of models by $2.98 \% \sim 8.35 \%$ ), AA (higher than the other four groups of models by percentage of $0.71 \% \sim 4.58 \%$ ) and Kappa $(1.91 \%$ 4.95\% higher than the other four groups of models) are better than other models, proving the feasibility of our proposed MARB-Net algorithm. Figure 3 visualizes the experimental results (using $40 \%$ of the samples as the training set for training). It can be clearly seen that the MARB-Net algorithm is superior to other models in denoising.

2) Results of the Pavia dataset: The characteristic of this dataset is that the number of samples of each type is large, but the shape and position of the samples are relatively scattered. It can be clearly seen from Table 3 that in $1 \%$ of the training samples, the Kappa coefficient of ATGIR is slightly higher than $0.14 \%$ of our proposed algorithm. The possible reason is that the algorithm constructs the relationship between deep features, but with the sample The increase in the number allows our proposed algorithm to obtain more deep features and make up for the relationship between the features, so the effect is better than the ATGIR algorithm. At the same time, the overall residual network experimental results are better than dense network and Alex-Net. Finally, at the moment when the training sample is $20 \%$, it can be clearly seen that the Kappa coefficient of our proposed MARB-Net algorithm is $0.3 \% \sim 1.58 \%$ higher than other models. It can be seen that the MARB-Net algorithm has a small amount of irregularities. The sample has better learning ability. Figure 4 shows the experimental results of the Pavia dataset (using 20\% of the samples as the training set for training).

3) Results of the Salinas data set: Salinas is a relatively standardized data set. Therefore, it can be seen from Table 4 that all models have obtained more accurate experimental results. The experimental results of residual network are better than dense network and Alex-Net as a whole. As the number of training samples increases, the recognition effect of MARB-Net algorithm is better. Obviously, the MARB-Net algorithm also has a good learning ability for regular data. Figure 5 shows the experimental results (using 20\% of the samples as the training set for training).

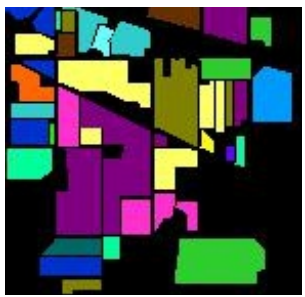

(a)

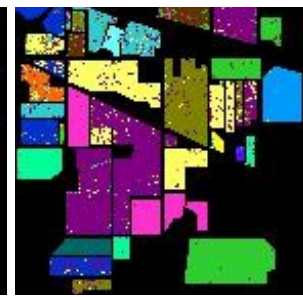

(b)

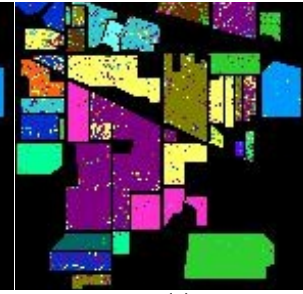

(c)

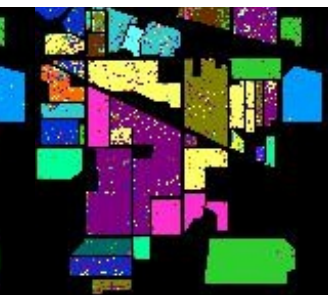

(d)
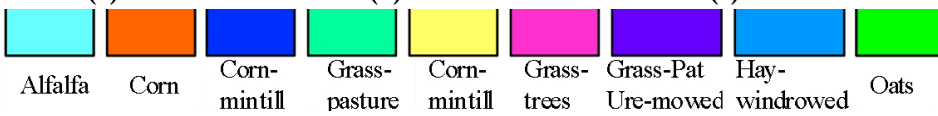

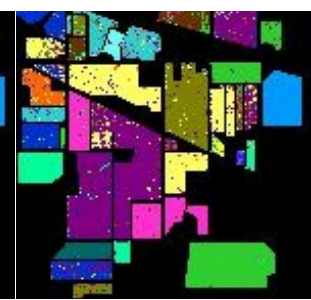

(e)

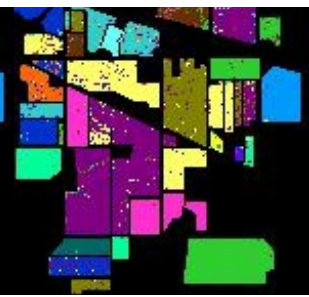

(f)

Fig.3. Visualization results of the Indian pines dataset. (a) Ground truth. (b) Res-Net. (c) Dense-Net. (d) Alex-Net. (e) ATGIR. (f) MARB-Net.

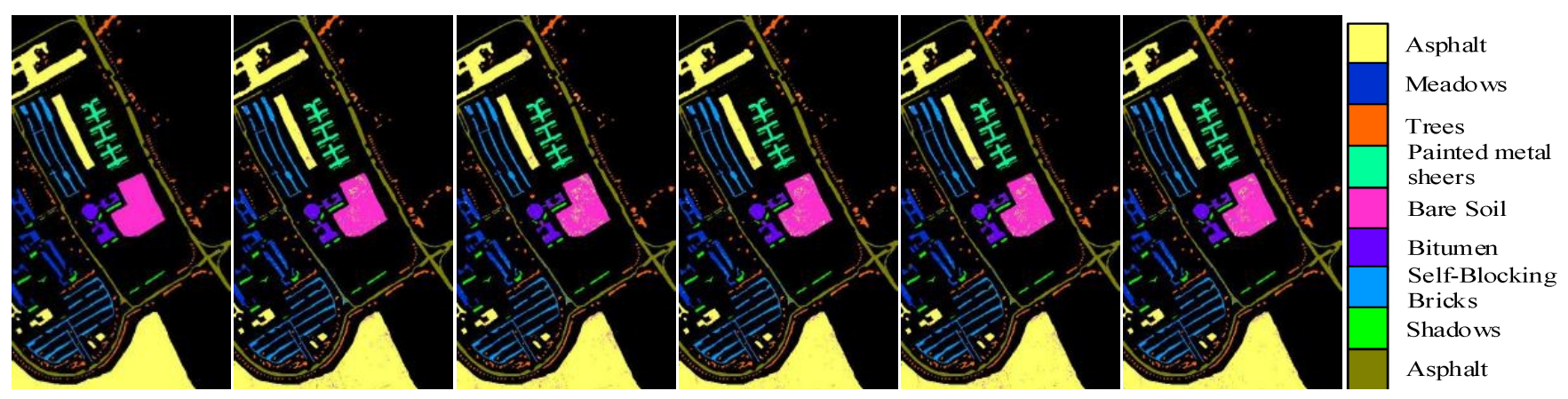

Fig.4. Visualization results of the University of Pavia dataset. (a) Ground truth. (b) Res-Net. (c) Dense-Net. (d) Alex-Net. (e) ATGIR. (f) MARB-Net. 


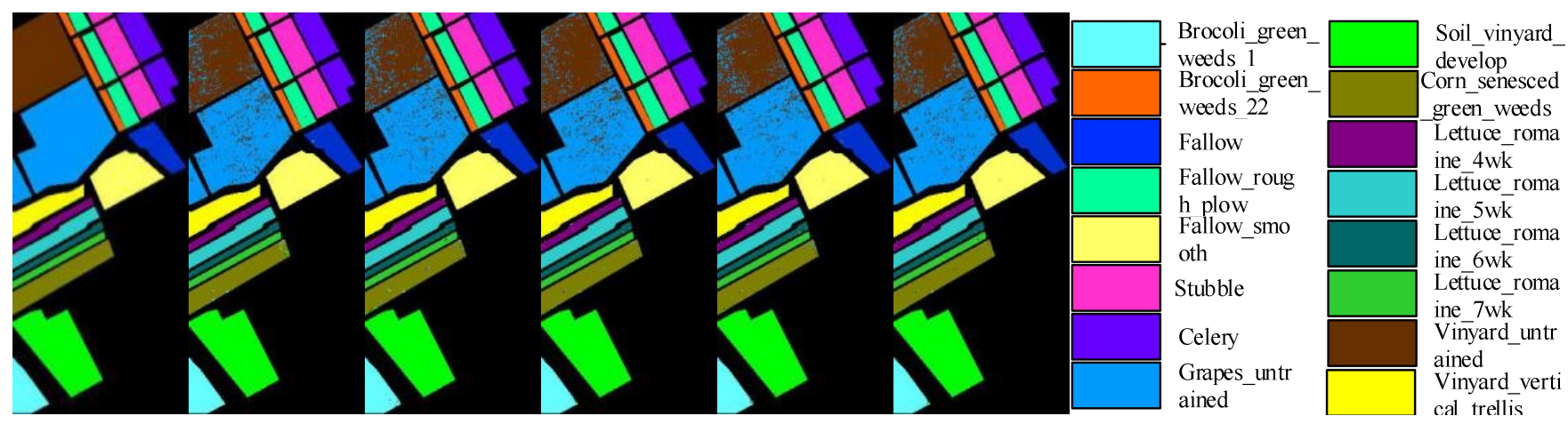

Fig.5. Visualization results of the Salinas dataset. (a) Ground truth. (b) Res-Net. (c) Dense-Net. (d) Alex-Net. (e) ATGIR. (f) MARB-Net.

\subsection{Ablation experiment of attention mechanism}

In this section, we mainly compare the effects of various attention mechanisms on the experimental results. We used the least divided training sample for each data set for testing (Indian Pines data set training used 5\% of the total sample, Pavia data set training used $1 \%$ of the total sample, Salinas data set training used the total sample 1\%). The number "1" represents the original attention mechanism, "2" represents the average pooled attention mechanism, and "3" represents the maximum pooled attention mechanism. Multiple numbers indicate that multiple different types of attention mechanisms are used at the same time.

Table 5 clearly shows that the effect of any attention mechanism model is better than that of models without attention mechanism. The combined experimental results of the two attention mechanism models are higher than the experimental results of the single attention mechanism model. At the same time, it can be clearly seen from the above table that the experimental results completed by the maximum pooling attention mechanism have the best effect, and the features generated by joint learning with other attention mechanisms are also more meaningful. This is because the maximum pooling attention mechanism is more prominent Local features with larger weights. At the same time, comparing the first set of experimental results in Table 5 with the Res-Net experimental models in each table, the experimental results AA and Kappa of our proposed model even without the attention mechanism are higher than those of the Res-Net model, so the two-way length The memory network strategy is also effective. Our MARB-Net algorithm optimizes the experimental results, so remote sensing image recognition based on multi-attention residual integrated network is feasible.

\subsection{Ablation experiment of different sub-models}

In this section, we mainly compare the effects of various sequence models on the experimental results. We used the least divided training sample for each data set for testing (Indian Pines data set training used $5 \%$ of the total sample, Pavia data set training used $1 \%$ of the total sample, Salinas data set training used the total sample $1 \%$ ).

Table 5 Experimental results of different attention mechanism algorithms on different data sets

\begin{tabular}{|c|c|c|c|c|c|c|c|c|c|}
\hline \multirow{2}{*}{ Methods } & \multicolumn{3}{|c|}{ Indian Pines } & \multicolumn{3}{c|}{ University of Pavia } & \multicolumn{3}{c|}{ Salinas } \\
\cline { 2 - 10 } & OA & AA & Kappa & OA & AA & Kappa & OA & AA & Kappa \\
\hline MARB_no_Attention & 0.7156 & 0.7107 & 0.6744 & 0.8530 & 0.8457 & 0.8020 & 0.8752 & 0.9259 & 0.8778 \\
\hline MARB_Attention_1 & 0.7228 & 0.7249 & 0.6814 & 0.8569 & 0.8513 & 0.8217 & 0.8982 & 0.9357 & 0.8866 \\
\hline MARB_Attention_2 & 0.7184 & 0.7195 & 0.6774 & 0.8534 & 0.8576 & 0.8254 & 0.8915 & 0.9263 & 0.8792 \\
\hline MARB_Attention_3 & 0.7180 & 0.7237 & 0.6838 & 0.8561 & 0.8531 & 0.8167 & 0.8944 & 0.9316 & 0.8822 \\
\hline MARB_Attention_1_2 & 0.7258 & 0.7294 & 0.6901 & 0.8770 & 0.8673 & 0.8352 & 0.9066 & 0.9384 & 0.8960 \\
\hline MARB_Attention_1_3 & 0.7419 & 0.7337 & 0.6953 & 0.8664 & 0.8622 & 0.8309 & 0.9035 & 0.9359 & 0.8925 \\
\hline MARB_Attention_2_3 & 0.7436 & 0.7297 & 0.6892 & 0.8699 & 0.8653 & 0.8283 & 0.9011 & 0.9362 & 0.8941 \\
\hline MARB & $\mathbf{0 . 7 4 6 1}$ & $\mathbf{0 . 7 3 7 5}$ & $\mathbf{0 . 6 9 7 8}$ & $\mathbf{0 . 8 7 6 3}$ & $\mathbf{0 . 8 7 0 6}$ & $\mathbf{0 . 8 3 4 1}$ & $\mathbf{0 . 9 1 2 6}$ & $\mathbf{0 . 9 4 8 3}$ & $\mathbf{0 . 9 0 0 1}$ \\
\hline
\end{tabular}

Table 6 Experimental results of different sub-model algorithms on different data sets

\begin{tabular}{|c|c|c|c|c|c|c|c|c|c|}
\hline \multirow{2}{*}{ Methods } & \multicolumn{3}{|c|}{ Indian Pines } & \multicolumn{2}{c|}{ University of Pavia } & \multicolumn{3}{c|}{ Salinas } \\
\cline { 2 - 10 } & OA & AA & Kappa & OA & AA & Kappa & OA & AA & Kappa \\
\hline AML_no_Bi-LSTM & 0.7227 & 0.7118 & 0.6824 & 0.8674 & 0.8474 & 0.8221 & 0.8529 & 0.8421 & 0.8359 \\
\hline AML_GRU & 0.7212 & 0.7295 & 0.6809 & 0.8569 & 0.8513 & 0.8217 & 0.9008 & 0.9281 & 0.8896 \\
\hline AML_Bi-GRU & 0.7152 & 0.7252 & 0.6740 & 0.8569 & 0.8494 & 0.8091 & 0.8873 & 0.9224 & 0.8745 \\
\hline AML_LSTM & 0.7410 & 0.7263 & 0.6845 & 0.8627 & 0.8521 & 0.8148 & 0.9106 & 0.9437 & 0.8925 \\
\hline MARB & $\mathbf{0 . 7 4 6 1}$ & $\mathbf{0 . 7 3 7 5}$ & $\mathbf{0 . 6 9 7 8}$ & $\mathbf{0 . 8 7 6 3}$ & $\mathbf{0 . 8 7 0 6}$ & $\mathbf{0 . 8 3 4 1}$ & $\mathbf{0 . 9 1 2 6}$ & $\mathbf{0 . 9 4 8 3}$ & $\mathbf{0 . 9 0 0 1}$ \\
\hline
\end{tabular}

Table 6 shows the experimental results of different sequence models. From the above experimental results, it can be clearly seen that after the depth features are integrated through the sequence model, the recognition ability of various remote sensing images is enhanced, which proves that the sequence model strategy is effective. At the same time, the two-way long and short-term memory network 
achieves the best effect in the model. Among them, the oneway GRU model is better than the two-way GRU in the three sets of data. The reason is that the GRU transfers the features directly to the next unit without any processing. Too many GRUs increase a lot of redundant features and therefore reduce Improved recognition accuracy. And LSTM has more memory cells, so it can retain more deep features, so it has better results. Therefore, the two-way long and short-term memory network has the best effect for the integration of deep features.

\section{Conclusion}

Hyperspectral images contain a large number of redundant features, making feature selection and mining more difficult, and making traditional image recognition models difficult to fully apply. To address the aforementioned issues, this paper proposes a multi-attention residual ensemble network, which not only selects features using residual multiple attention mechanism models, but also fully excavates a large number of deep features using the residual network and implements it using a Bi-LSTM network. The above issues are wellsolved by integrating in-depth features. Three public data sets produced the best results. The feasibility of the MARBNet algorithm has been fully demonstrated. The next step in our research will be to see how we can improve recognition results while lowering parameters and increasing computational efficiency.

\section{Acknowledgement}

This work was supported by the Hunan Key Laboratory of Intelligent Logistics Technology under Grant 2019TP1015.

\section{References}

[1] Su, H., Yang, X., \& Yan, X. H. (2019, July). Estimating Ocean Subsurface Salinity from Remote Sensing Data by Machine Learning. In IGARSS 2019-2019 IEEE International Geoscience and Remote Sensing Symposium (pp. 8139-8142).

[2] You, H., Tian, S., Yu, L., \& Lv, Y. (2019). Pixel-level remote sensing image recognition based on bidirectional word vectors. IEEE Transactions on Geoscience and Remote Sensing, 58(2), 1281-1293.

[3] Shumilo, L., Yailymov, B., Kussul, N., Lavreniuk, M., Shelestov, A., \& Korsunska, Y. (2019, April). Rivne City land cover and land surface temperature analysis using remote sensing data. In 2019 IEEE 39th international conference on electronics and nanotechnology (ELNANO) (pp. 813-816).

[4] You, J., Li, X., Low, M., Lobell, D., \& Ermon, S. (2017, February). Deep gaussian process for crop yield prediction based on remote sensing data. In Proceedings of the AAAI Conference on Artificial Intelligence (Vol. 31, No. 1).

[5] Wu, S., \& Chen, H. (2020). Smart city oriented remote sensing image fusion methods based on convolution sampling and spatial transformation. Computer Communications, 157, 444-450.
[6] Ge, Y., Jiang, S., Xu, Q., Jiang, C., \& Ye, F. (2018). Exploiting representations from pre-trained convolutional neural networks for high-resolution remote sensing image retrieval. Multimedia Tools and Applications, 77(13), 17489-17515.

[7] El-Shafie, A. H. A., Zaki, M., \& Habib, S. E. D. (2019, June). Fast CNN-based object tracking using localization layers and deep features interpolation. In 2019 15th International Wireless Communications \& Mobile Computing Conference (IWCMC) (pp. 1476-1481).

[8] Xie, F., Li, F., Lei, C., Yang, J., \& Zhang, Y. (2019). Unsupervised band selection based on artificial bee colony algorithm for hyperspectral image classification. Applied Soft Computing, 75, 428-440.

[9] Zhou, Y., \& Wang, M. (2019, July). Remote sensing image classification based on AlexNet network model. In international conference on frontier computing (pp. 913-918).

[10] Zhang, J., Lu, C., Li, X., Kim, H. J., \& Wang, J. (2019). A full convolutional network based on DenseNet for remote sensing scene classification. Math. Biosci. Eng, 16(5), 3345-3367.

[11] Bahdanau, D., Cho, K., \& Bengio, Y. (2014). Neural machine translation by jointly learning to align and translate. arXiv preprint arXiv: 1409.0473.

[12] Hang, R., Liu, Q., Hong, D., \& Ghamisi, P. (2019). Cascaded recurrent neural networks for hyperspectral image classification. IEEE Transactions on Geoscience and Remote Sensing, 57 (8), 5384-5394.

[13] Chu, R. S., Ng, H. C., Wang, X., \& Luk, W. (2019, July). Convolution Based Spectral Partitioning Architecture for Hyperspectral Image Classification. In IGARSS 2019-2019 IEEE International Geoscience and Remote Sensing Symposium (pp. 3962-3965).

[14] Paoletti, M. E., Haut, J. M., Plaza, J., \& Plaza, A. (2019, July). Solving Deep Neural Networks with Ordinary Differential Equations for Remotely Sensed Hyperspectral Image Classification. In IGARSS 2019-2019 IEEE International Geoscience and Remote Sensing Symposium (pp. 576-579).

[15] Chen, Y., Wan, J., Zhang, J., Zhao, J., Ye, F., Wang, Z., \& Liu, S. (2019, July). Automatic Extraction Method of Sargassum Based on Spectral-Texture Features of Remote Sensing Images. In IGARSS 2019-2019 IEEE International Geoscience and Remote Sensing Symposium (pp. 3705-3707).

[16] Ma, W., Pan, Z., Guo, J., \& Lei, B. (2019). Achieving superresolution remote sensing images via the wavelet transform combined with the recursive res-net. IEEE Transactions on Geoscience and Remote Sensing, 57(6), 3512-3527.

[17] Zhang, C., Han, M., \& Xu, M. (2018, July). Multi-feature Classification of Hyperspectral Image via Probabilistic SVM and Guided Filter. In 2018 International Joint Conference on Neural Networks (IJCNN) (pp. 1-7).

[18] Tang, Y., Li, L., Wang, C., Chen, M., Feng, W., Zou, X., \& Huang, K. (2019). Real-time detection of surface deformation and strain in recycled aggregate concrete-filled steel tubular columns via four-ocular vision. Robotics and Computer-Integrated Manufacturing, 59, 36-46.

\section{Biographies}


Weiwei Cai is currently practicing at the Laboratory of Artificial Neural Networks and High Speed Circuits, Institute of Semiconductors, Chinese Academy of Sciences, Prior to that, he worked with IT industry for more than ten years in the roles of an System Architect and the Program Manager. His research interests include machine learning, deep learning, and computer vision.

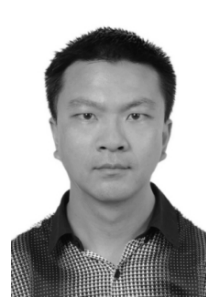

Zhanguo Wei received the Ph.D. degree from the School of Technology, Beijing Forestry University, Beijing, China. He has been working as an Associate Professor with the Central South University of Forestry and Technology, Changsha, China, since 2011. His research interests include information retrieval, data mining, big data, and deep learning.

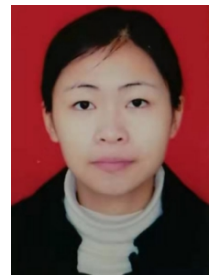

Runmin Liu received the B.A. degree in English from Hunan Agricultural University, Changsha, China, in 2006. She is currently working toward the M.S. degree in Sports Engineering from Wuhan Sports University, Wuhan, China. Her research interests include computer vision, deep learning, and image processing.

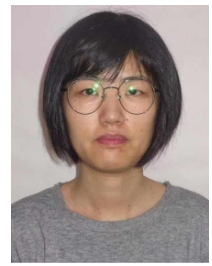

Yuan Zhuang is currently pursuing the master's degree with the Central South University of Forestry and Technology, Changsha, China. Her main research include deep learning and data mining.

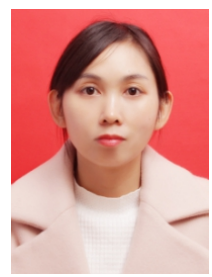

Yan Wang is currently working the Changsha Astra Information Technology Co., Ltd. Changsha, China. Her main research interests include neural networks and computer vision.

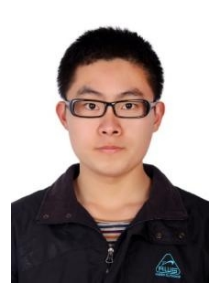

Xin Ning received the B.S. degree in software engineering in 2012, and the Ph.D. degree in electronic circuit and system from university of Chinese Academy of Sciences, in 2017. He is currently an Associate Professor with the Laboratory of Artificial Neural Networks and High Speed Circuits, Institute of Semiconductors, Chinese Academy of Sciences. He has published by first or corresponding author more than 40 papers in journals and refereed conferences. His current research interests include pattern recognition, computer vision, and image processing. 